\title{
The AFD Sensory Neurons Encode Multiple Functions Underlying Thermotactic Behavior in Caenorhabditis elegans
}

\author{
Damon A. Clark, ${ }^{1}$ David Biron, ${ }^{1,2}$ Piali Sengupta, ${ }^{2}$ and Aravinthan D. T. Samuel ${ }^{1}$ \\ ${ }^{1}$ Department of Physics, Harvard University, Cambridge, Massachusetts 02138, and ${ }^{2}$ Department of Biology and National Center for Behavioral Genomics, \\ Brandeis University, Waltham, Massachusetts 02454
}

\begin{abstract}
The thermotactic behaviors of Caenorhabditis elegans indicate that its thermosensory system exhibits exquisite temperature sensitivity, long-term plasticity, and the ability to transform thermosensory input into different patterns of motor output. Here, we study the physiological role of the AFD thermosensory neurons by quantifying intracellular calcium dynamics in response to defined temperature stimuli. We demonstrate that short-term adaptation allows AFD to sense temperature changes as small as $0.05^{\circ} \mathrm{C}$ over temperature ranges as wide as $10^{\circ} \mathrm{C}$. We show that a bidirectional thermosensory response (increasing temperature raises and decreasing temperature lowers the level of intracellular calcium in AFD) allows the AFD neurons to phase-lock their calcium dynamics to oscillatory thermosensory inputs. By analyzing the thermosensory response of AFD dendrites severed from their cell bodies by femtosecond laser ablation, we show that long-term plasticity is encoded as shifts in the operating range of a putative thermoreceptor(s) in the AFD sensory endings. Finally, we demonstrate that AFD activity is directly coupled to stimulation of its postsynaptic partner AIY. These observations indicate that many functions underlying thermotactic behavior are properties of one sensory neuronal type. Encoding multiple functions in individual sensory neurons may enable $C$. elegans to perform complex behaviors with simple neuronal circuits.
\end{abstract}

Key words: C. elegans; behavior; thermotaxis; calcium [Ca]; thermosensation; laser axotomy

\section{Introduction}

A major goal of systems neuroscience is to understand how layers of neural circuitry transform complex sensory inputs into precise motor outputs. Only in a few cases of simple reflexive behaviors have entire pathways been mapped from sensory to motor neurons (e.g., the tail-flip response in fish or touch avoidance in nematodes) (Furshpan and Furukawa, 1962; Chalfie et al., 1985). In comparison, the neural circuits that mediate the ability of animals to explore variable environments are poorly understood, because they perform more sophisticated transformations of sensory inputs into directed movements. Caenorhabditis elegans provides an opportunity to map exploratory navigational behaviors to a wiring diagram in which only a few layers of interneurons separate the neurons at the sensory periphery from the motor neurons that direct movement (White et al., 1986). Systematic analyses using defined neuronal lesions in C. elegans have mapped neurons that mediate exploratory movements in isotropic environments (Tsalik and Hobert, 2003; Wakabayashi et al., 2004; Gray et al., 2005). However, mapping the more sophisti-

Received March 16, 2006; revised June 5, 2006; accepted June 6, 2006.

This work was supported by National Institutes of Health Grant P01 NS44232 (P.S.). D.A.C. was supported by the National Science Foundation (NSF), D.B. was supported by the Human Frontiers Science Program, and A.D.T.S. was supported by the Sloan Foundation, the McKnight Foundation, and the NSF. We thank Keng-hui Lin for advice on ITO heating, Koutarou Kimura for advice regarding the strain Ex1518, and Mayumi Shibuya and Sara Wasserman for assistance with genetics and molecular biology.

Correspondence should be addressed to Dr. Aravinthan D. T. Samuel at the above address. E-mail: samuel@physics.harvard.edu.

DOI:10.1523/JNEUROSCI.1137-06.2006

Copyright $\odot 2006$ Society for Neuroscience $\quad$ 0270-6474/06/267444-08\$15.00/0 cated sensorimotor transformations that mediate directed movement in variable environments is a larger challenge.

The thermotactic behaviors of C. elegans allow the animal to navigate spatial thermal gradients in an experience-dependent manner (Hedgecock and Russell, 1975). C. elegans stores a thermotactic set-point $\left(\mathrm{T}_{\mathrm{S}}\right)$ corresponding to its previous cultivation temperature. When navigating thermal gradients at temperatures above $T_{S}$, C. elegans crawls toward colder temperatures, exhibiting what is called cryophilic movement. When navigating thermal gradients near $\mathrm{T}_{S}$, C. elegans crawls along isotherms, deviating from each isotherm by as little as $0.1^{\circ} \mathrm{C}$ (Hedgecock and Russell, 1975; Ryu and Samuel, 2002). Therefore, the thermosensory system of C. elegans has multiple functions: (1) it sets the temperature range of its thermotactic behaviors on the basis of experience; (2) it exhibits high sensitivity to temperature gradients over these broad temperature ranges; and (3) it enacts different strategies to transform thermosensory input into motor output in the different temperature ranges. In principle, these functions could be performed by multiple sensory neurons or by progressive transformations of sensory information by downstream neural circuits.

To date, only the AFD neurons have been identified with a thermosensory role in C. elegans; genetic and physical perturbations of AFD disrupt thermotactic behavior (Mori and Ohshima, 1995; Satterlee et al., 2001). Kimura et al. (2004) showed that AFD responds to warming ramps at temperatures above $T_{S}$, suggesting that the AFD neurons themselves store a memory of the $\mathrm{T}_{\mathrm{S}}$. Here, we image $\mathrm{Ca}^{2+}$ dynamics in the AFD neurons in response to defined thermal stimuli and show that AFD is endowed with high temperature sensitivity, short-term adaptation, and long-term 
plasticity and also acts as a filter to amplify oscillating thermosensory input. We show that $\mathrm{Ca}^{2+}$ dynamics can be resolved in individual neuronal compartments: at the AFD sensory endings; at the AFD soma; and at the axons of the AIY interneuron triggered by AFD synaptic output. Our results suggest that many functions required for thermotactic behavior are performed by one sensory neuronal type. This observation may explain how complex sensorimotor behaviors in C. elegans are condensed in simple neural circuits.

\section{Materials and Methods}

Cultivation. Worms were cultivated with OP50 bacterial food using standard procedures (Brenner, 1974). The strain expressing cameleon in the AFD neurons, kdkEx1518 [H13p::yc2.12], was a gift from I. Mori (Nagoya University, Nagoya, Japan) and was used in all AFD imaging experiments. Cameleon was expressed in the AIY interneurons by driving expression of a cameleon cDNA (yc2.12) under the C. elegans ttx-3 promoter.

Temperature stimulus. For each calcium imaging experiment, a young adult worm was glued to a thin agar pad with cyanoacrylate glue (Abbott Laboratories, Abbott Park, IL), and a small $200 \mu \mathrm{m}$ thermocouple (Physitemp Instruments, Clifton, NJ) was placed within $3 \mathrm{~mm}$ of the worm on the agar surface. A coverslip was placed over the worm and thermocouple, and the agar pad was placed on the glass slide of a temperature-controlled apparatus. The glass slide was coated with transparent indium tin oxide (ITO) (Delta Technologies, Stillwater, MN) and was attached to a brass plate cooled by internally circulated temperaturecontrolled water. The brass plate was set to a cold base temperature, while electrical current through the ITO warmed the slide and the sample to a specified temperature. The current was under computer control, using a proportional-integral-derivative feedback loop programmed in LabVIEW (National Instruments, Austin, TX) using readout from the thermocouple near the worm. The sample was shielded from electric fields using a second layer of ITO between the current-carrying layer and the sample, and by grounding the microscope objective. Using this system, we could achieve temperature stability $\left(<0.01^{\circ} \mathrm{C} \mathrm{rms} \mathrm{deviation)}\right.$ and controlled heating and cooling as fast as $10^{\circ} \mathrm{C} / \mathrm{min}$ to create stimulus waveforms such as steps and pulses with minimal overshoots.

When measuring the threshold temperature of worms cultivated at 15 , 20 , or $25^{\circ} \mathrm{C}$, we imaged worms within $10 \mathrm{~min}$ of placing the worms on the microscope, except when surgery was performed, in which case the second measurement was within $30 \mathrm{~min}$ of placing the worms on the microscope. All surgical preparations and imaging experiments were conducted in an $18^{\circ} \mathrm{C}$ room, so that all worms experienced the same exposure temperatures between removal from their incubators and activation of the temperature-controlled stage. In the experiments shown in Figures 1, 5, and 6, we measured the lower bound of the operating range using a sine-wave temperature oscillation, with typical peak-to-peak amplitudes between 0.1 and $0.3^{\circ} \mathrm{C}$ and periods between 15 and $20 \mathrm{~s}$, added to a positive linear ramp of $0.01^{\circ} \mathrm{C} / \mathrm{s}$ in repeated trials lasting $\sim 3 \mathrm{~min}$ each. For other experiments, we found that worms exhibited measurable calcium dynamics for up to $45 \mathrm{~min}$ after being glued. We did not observe any differences in calcium dynamics between the AFDL and AFDR neurons, so we typically focused on the neuron closest to the objective.

Imaging. During the temperature stimulus, the fluorescence of cameleon expressed in the AFD and AIY neurons was imaged using a Nikon (Tokyo, Japan) $40 \times$ air objective [0.95 numerical aperture (NA)] and a Photometrics (Tucson, AZ) CoolSNAP camera. We used a Nikon $100 \times$ oil objective (1.4 NA) for imaging calcium responses in femtosecond laser ablation experiments and for all measurements of calcium dynamics in the AFD sensory endings. The yellow and cyan emissions of cameleon were split using dichroic mirrors and bandpass filters (Chroma, Rockingham, VT), such that two images of different wavelengths were simultaneously projected onto two halves of one video frame. Fluorescence resonance energy transfer (FRET) ratios were computed for each video frame by selecting the region of interest surrounding the AFD neuron (which was unambiguously the brightest object in the field of view), averaging the intensities of the brightest pixels from the region in each frame (where the number of pixels matched the area of the neuron), and calculating the ratio of the intensities of the yellow and cyan channels. By identifying the region of interest in each frame, we minimized artifacts attributable to $x-y$ motion of the neuron. In some data records, small changes in the fluorescence of the yellow and cyan channel exhibited covariance, which might be attributed to $z$-motions of the neuron that did not measurably affect the ratio of the fluorescence intensities (Kerr et al., 2000). Exposure times to excitation light ranged from 250 to $600 \mathrm{~ms}$ at a $1 \mathrm{~Hz}$ frame rate. The choice of exposure times and frame rate was a balance between increasing signal to noise in the measurement represented by each video frame and minimizing measurement variation between frames attributable to photobleaching. Images were analyzed using MATLAB (Mathworks, Natick, MA).

Surgery. Femtosecond laser ablation was performed as described in detail previously (Shen et al., 2005). In brief, laser pulses, $800 \mathrm{~nm}$ in wavelength and $100 \mathrm{fs}$ in duration, were produced by a cavity-dumped Ti:sapphire laser (KMLabs, Boulder, CO). A $1 \mathrm{kHz}$ pulse train was tightly focused using the Nikon $100 \times(1.4 \mathrm{NA})$ objective that was used for FRET imaging in these experiments. We targeted the AFD dendrites or soma for ablation using fluorescence microscopy. When severing AFD dendrites, we used $\sim 5-15 \mathrm{~nJ}$ pulses at the sample, which is above the threshold of vaporization (Shen et al., 2005). When killing the AFD soma, we targeted the nucleus and used $\sim 15-20 \mathrm{~nJ}$ pulses, until the soma was visibly destroyed.

\section{Results}

\section{The operating range of AFD spans the temperature range of} isothermal tracking and cryophilic movement

Kimura et al. (2004) showed that the AFD neuron responds to warming ramps at temperatures above the previous cultivation temperature of the worm $\left(\mathrm{T}_{\mathrm{S}}\right)$ by monitoring intracellular $\mathrm{Ca}^{2+}$ dynamics using the $\mathrm{Ca}^{2+}$-binding fluorescent protein cameleon (Miyawaki et al., 1999). First, we sought to quantify the operating range of the AFD neuron. At temperatures above a certain lower bound, we found that AFD intracellular $\mathrm{Ca}^{2+}$ dynamics become strongly phase-locked to a sinusoidal temperature oscillation; at temperatures below the lower bound, $\mathrm{Ca}^{2+}$ dynamics are not correlated with the sinusoidal temperature oscillation (Fig. 1A). We were able to quantify the lower bound of the operating range of the AFD neuron by subjecting worms to a positive linear temperature ramp. For worms cultivated at $20^{\circ} \mathrm{C}$, the linear ramp begins to stimulate an increase in intracellular $\mathrm{Ca}^{2+}$ concentration in the AFD neuron when it reaches $\sim 17^{\circ} \mathrm{C}$ (Fig. $1 \mathrm{~B}$ ). This is consistent with the observations by Kimura et al. (2004), who observed increases in the levels of intracellular $\mathrm{Ca}^{2+}$ in response to temperature ramps above the $\mathrm{T}_{\mathrm{S}}$. Based on these observations, we were able to simultaneously measure the lower bound of the AFD operating range and monitor the phase-locking of AFD $\mathrm{Ca}^{2+}$ dynamics to the thermosensory input by using a temperature waveform consisting of a rapid sinusoidal oscillation added to a positive linear ramp (Fig. $1 B$ ). We found that the lower bound of the operating range of AFD is near 15,17 , and $21^{\circ} \mathrm{C}$ for worms cultivated at 15,20 , and $25^{\circ} \mathrm{C}$, respectively (Fig. $1 \mathrm{C}$ ), and is thus closely related to their $\mathrm{T}_{\mathrm{S}}$. Because worms track isotherms within $\pm 2^{\circ}$ of their previous cultivation temperature (Ryu and Samuel, 2002), the lower bound of the operating range of AFD in animals grown at specific temperatures is near the lowest temperature at which they would track isotherms. Worms also exhibit cryophilic movement at temperatures higher than $\mathrm{T}_{\mathrm{S}}$. Because intracellular $\mathrm{Ca}^{2+}$ dynamics in AFD continues to warmer temperatures, the operating range of the AFD neuron appears to overlap both the temperature range in which C. elegans tracks isotherms and the temperature range in which it displays cryophilic movement. 
A

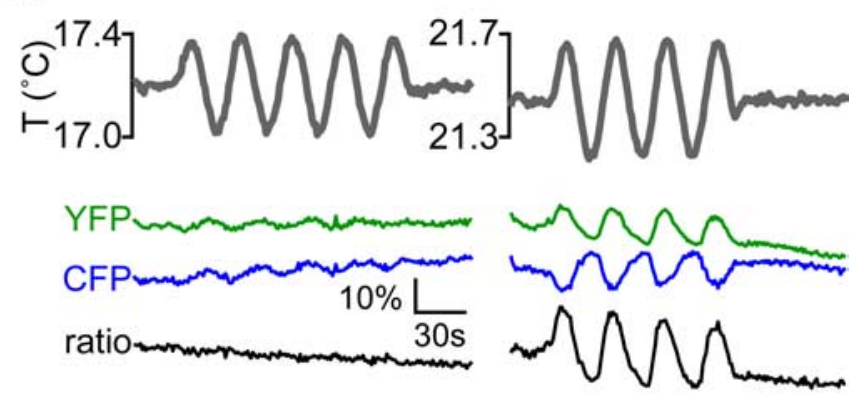

B
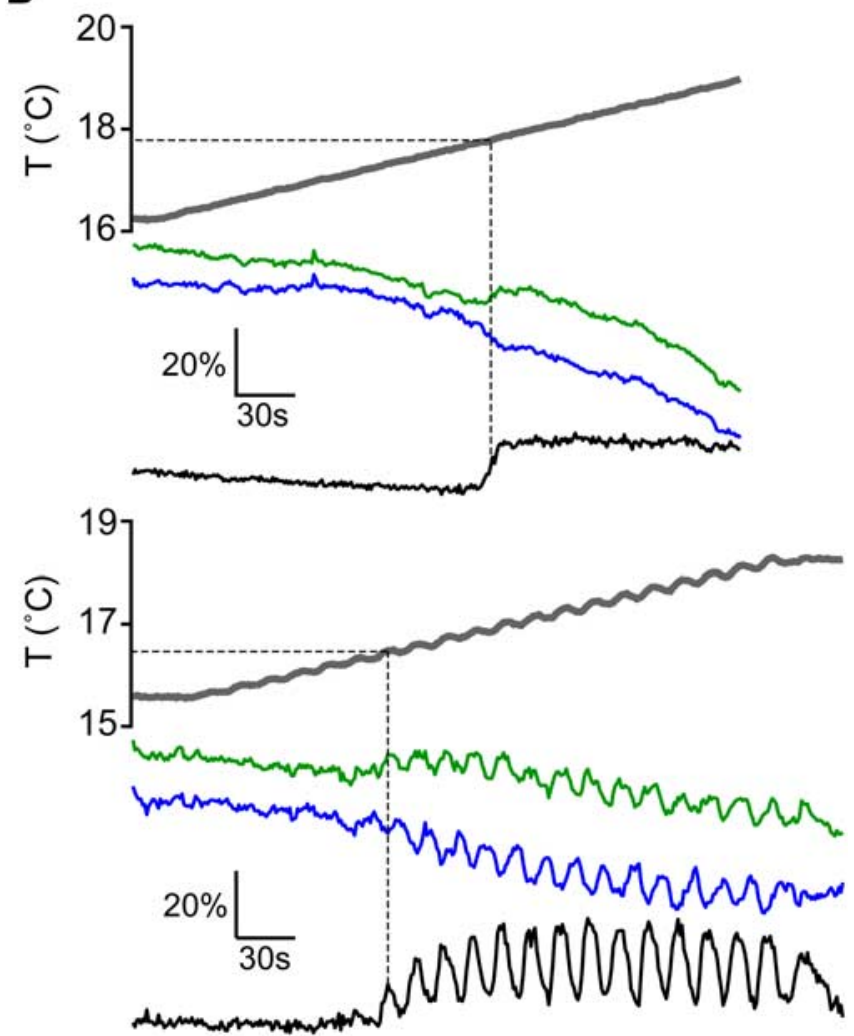

C

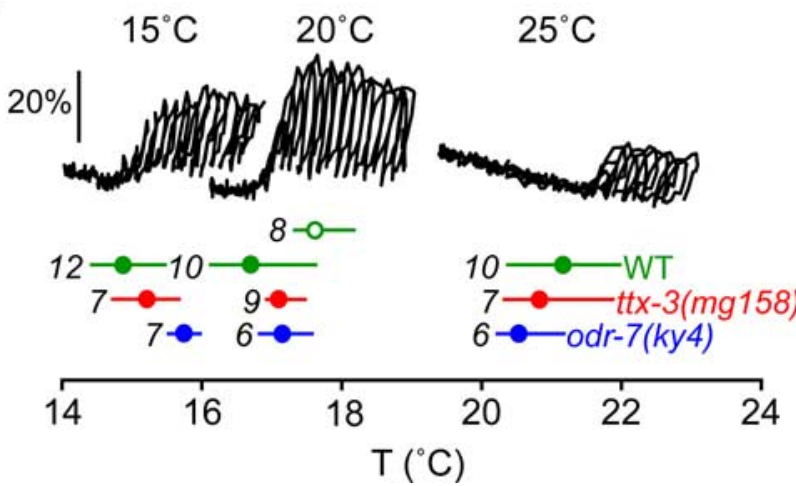

Figure 1. Operating range of $\mathrm{AFD} \mathrm{Ca}{ }^{2+}$ dynamics. $A$, Worms cultivated at $20^{\circ} \mathrm{C}$ were subjected to sinusoidal temperature variations (gray line) while simultaneously monitoring fluorescence emission at the AFD soma from cameleon in the yellow fluorescent protein (YFP) emission channel (green line) and cyan fluorescent protein (CFP) emission channel (blue line). The FRET response (black line) is calculated as the ratio of yellow and cyan emission. At temperatures $>2-3^{\circ} \mathrm{C}$ below $T_{s}$, the fluorescence of cameleon is unaffected by temperature changes, indicating an absence of intracellular $\left[\mathrm{Ca}^{2+}\right]$ changes. At temperatures near or above $\mathrm{T}_{\mathrm{S}}$, the
Mutations that affect synaptic partners do not affect AFD $\mathrm{Ca}^{2+}$ dynamics

The AWA sensory neurons and the AIY interneurons are the primary presynaptic and postsynaptic partners of the AFD neurons, respectively (White et al., 1986). To determine whether these neurons contributed to AFD thermosensory responses, we quantified AFD intracellular $\mathrm{Ca}^{2+}$ dynamics in worms carrying a mutation in the odr-7 nuclear hormone receptor gene, which alters the cell fate of the AWA neurons, and in worms carrying a mutation in the ttx-3 LIM homeobox gene, which disrupts the development of the AIY neurons (Sengupta et al., 1994; Hobert et al., 1997). $\mathrm{Ca}^{2+}$ responses of the AFD neurons were unaltered in either $o d r-7(k y 4)$ or $t t x-3(m g 158)$ mutants (Fig. 1C), such that the operating range of the AFD neurons of either mutant was similar to that of wild-type worms. We note that the thermosensory behaviors of $t t x-3$ mutants appear to be independent of previous experience such that these worms do not display isothermal tracking near $\mathrm{T}_{\mathrm{S}}$ and display cryophilic movement at both $\mathrm{T}>\mathrm{T}_{\mathrm{S}}$ and $\mathrm{T}<\mathrm{T}_{\mathrm{s}}$. Nevertheless, a physiological correlate of $\mathrm{T}_{\mathrm{S}}$, the lower bound of the operating range of AFD, is intact in ttx-3 mutants. Although the experience-dependent operating range of AFD is unaltered in $t t x-3$ mutants, we suggest that disruption of AIY prevents this information from modulating the rest of the thermosensory circuit or overall behavior (Fig. 1C).

\section{AFD is sensitive to $0.05^{\circ} \mathrm{C}$ temperature changes}

During isothermal tracking, C. elegans is able to stay within $\pm 0.1^{\circ} \mathrm{C}$ of an isotherm for several minutes, which indicates a robust sensitivity to small temperature changes (Hedgecock and Russell, 1975; Ryu and Samuel, 2002). To determine whether AFD is sufficiently sensitive to play a role in active thermotactic movement, we measured the dynamic range of the AFD thermosensory response. We exposed worms to a stimulus waveform consisting of a sinusoidal oscillation multiplied by a linear ramp (Fig. 2A). With this stimulus, the FRET signal of cameleon in AFD becomes strongly phase-locked to the stimulus waveform but saturates when the size of the temperature variations reaches $\sim 0.4^{\circ} \mathrm{C}$ change in $15 \mathrm{~s}$ (Fig. $2 \mathrm{~B}$ ). This saturation could correspond to physiologically meaningful saturation of $\mathrm{Ca}^{2+}$ levels or to saturation of the cameleon indicator. Wherever the saturation point, we demonstrate that the AFD thermosensory response is 
A
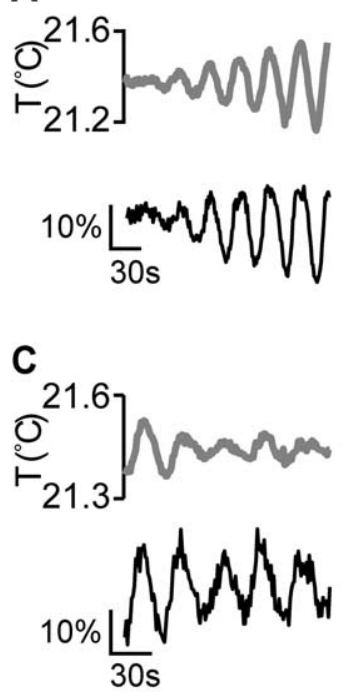

Figure 2. Dynamic range and sensitivity of the AFD response. $\boldsymbol{A}$, Wild-type worms were subjected to temperature stimuli within the AFD operating range consisting of a 30 s period sine wave multiplied by a linear ramp. The size of the FRET signal increases with the amplitude of the sine wave. $\boldsymbol{B}$, The peak-to-peak fractional change in the FRET signal is plotted against the peak-to-peak temperature change of the stimulus used in $A$. Three representative signals from individual worms are shown by dashed lines. Mean \pm 1 SEM error bars calculated from 21 worms are shown by circles connected by the thick black line. The FRET signal saturation corresponds to temperature changes of $\sim 0.4^{\circ} \mathrm{C}$ in $15 \mathrm{~s}$. C, FRET signals were apparent well below saturation. Here, we show a $10 \%$ FRET signal in an AFD soma stimulated by a temperature change of $\sim 0.05^{\circ} \mathrm{C}$ in $15 \mathrm{~s}$.

within the physiological range of thermotactic movement because we measured robust $\mathrm{Ca}^{2+}$ responses in AFD with temperature changes as small as $0.05^{\circ} \mathrm{C}$ in $15 \mathrm{~s}$ (Fig. 2C). Therefore, AFD is sufficiently sensitive to detect the temperature changes that accompany isothermal tracking movements.

\section{The AFD response is bidirectional and adaptive}

Next, we exposed worms to different temperature waveforms and quantified the temporal dynamics of the FRET response. We found that sinusoidal stimulus waveforms with periods between 10 and $240 \mathrm{~s}$ lead to phase-locked FRET responses with measurable phase differences (we were unable to explore the effects of shorter or longer stimulus periods because of experimental factors related to the stimulus delivery system and to the limited recording times of each experiment). The amount of the phase difference between stimulus and response waveforms depends on the period of the sine-wave stimulus and appears to gradually decrease with shorter stimulus periods. Therefore, with a short stimulus period, the response waveform seems to follow the stimulus waveform with negligible phase difference (Fig. 3A).

To more fully characterize the transformation between sensory input and AFD neuronal response, we measured intracellular $\mathrm{Ca}^{2+}$ dynamics in response to temperature steps. We found that the FRET response to temperature steps is bidirectional and adaptive. A temperature upstep evokes an immediate increase in the level of intracellular $\mathrm{Ca}^{2+}$ (within $<2 \mathrm{~s}$ of stimulus onset), which returns to the prestimulus level after 15-20 s. A temperature downstep evokes an immediate decrease in the level of intracellular $\mathrm{Ca}^{2+}$, but with a slower return to the prestimulus level, not measurable within $80 \mathrm{~s}$ (Fig. 3B). The measurable response to a temperature downstep suggests that the steady-state level of intracellular $\mathrm{Ca}^{2+}$ in AFD is above the lowest level of intracellular
$\mathrm{Ca}^{2+}$ that AFD can achieve. The asymmetric recovery rates after temperature upsteps and downsteps affect the response pattern to successive temperature pulses of opposite sign. For example, if a positive temperature pulse is followed by a negative temperature pulse, AFD intracellular $\mathrm{Ca}^{2+}$ dynamics tracks the rising and falling phases of the first pulse, fails to respond to the falling phase of the second pulse, and rises with the rising phase of the second pulse. However, if a negative temperature pulse is followed by a positive temperature pulse, AFD intracellular $\mathrm{Ca}^{2+}$ dynamics tracks the falling and rising phases of the first pulse, is allowed to return to the prestimulus level, and is able to track both the rising and falling phases of the second pulse (Fig. $3 C$ ). The asymmetric rates of adaptation after upsteps and downsteps also affect the response to successive steps of the same sign. With successive upsteps separated by $45 \mathrm{~s}$, the significant recovery of the level of intracellular $\mathrm{Ca}^{2+}$ after each step allows a strong response to the succeeding step. With successive downsteps separated by $45 \mathrm{~s}$, slow recovery means that the level of intracellular $\mathrm{Ca}^{2+}$ hits a floor after the first downstep that inhibits responses thereafter (Fig. 3D).

\section{AFD temperature detection and storage of $T_{S}$ occur at its sensory endings}

The thermosensitive element in AFD has not yet been identified but is likely to be localized to the sensory endings of the AFD dendrites, along with the cyclic nucleotide-gated channels encoded by the genes $\operatorname{tax}-2$ and $\operatorname{tax}-4$, which are required for thermosensory behavior (Coburn and Bargmann, 1996; Komatsu et al., 1996). We found that we could reliably measure FRET responses to sinusoidal temperature waveforms from the AFD sensory endings. It is possible to snip nerve fibers in C. elegans using tightly focused femtosecond laser pulses (Yanik et al., 2004; Chung et al., 2006). We found that snipping an AFD dendrite midway between the soma and the sensory endings (Fig. $4 A$ ) does not disrupt $\mathrm{Ca}^{2+}$ dynamics measured at the sensory endings (Fig. $4 B, C)$. In most cases, $\mathrm{Ca}^{2+}$ dynamics in an AFD soma were abolished after the ipsilateral AFD dendrite was snipped (Fig. $4 B$ ). In some cases, however, we measured normal $\mathrm{Ca}^{2+}$ dynamics in an AFD soma after its own dendrite was severed. AFDL and AFDR neurons are interconnected by a gap junction in the nerve ring, so we suggest that these cases are as a result of electrical synaptic transmission that spreads the output of one sensory dendrite to both neurons (Fig. 4C). Indeed, in these cases, we found that we could abolish somatic $\mathrm{Ca}^{2+}$ dynamics by subsequently severing the dendrite of the contralateral AFD neuron (Fig. 4C). The variability in AFD activity after severing just one dendrite might suggest worm-to-worm variability in the gap junction coupling between the two AFD neurons. We note that in these measurements of AFD $\mathrm{Ca}^{2+}$ dynamics, it was not possible to verify that only the AFD dendrite was snipped by exposure to the femtosecond laser pulses without damaging neighboring nerve fibers; however, we used the same parameters for femtosecond laser ablation in these measurements as we used in a recent study, in which we snipped the AFD dendrites without disrupting the function of the nearby dendrites of the ASH osmosensory neurons (Chung et al., 2006).

Using sinusoidal stimulus waveforms, we found the same phase difference between temperature stimulus and FRET response in the sensory endings as we previously measured in the soma (2.2 s for a $30 \mathrm{~s}$ period) (Fig. $5 A$ ). Therefore, $\mathrm{Ca}^{2+}$ dynamics throughout the AFD neuron are evoked with near simultaneity, which suggests rapid propagation of a thermosensory signal throughout the cell. The most likely explanation is that mechanisms for $\mathrm{Ca}^{2+}$ entry and clearance from the AFD cytoplasm are 
directly coupled to changes in AFD cell membrane potential. Direct evidence that the thermotactic set-point is stored in part at the sensory endings was provided by femtosecond laser ablation. Severing the AFD dendrite does not significantly affect the operating range of the sensory endings (Fig. 5B). The lower bound of the operating range of the sensory endings of severed AFD dendrites is near 16,18 , and $21^{\circ} \mathrm{C}$ for worms cultivated at 15,20 , and $25^{\circ} \mathrm{C}$, respectively (Fig. $5 C$ ), and is thus closely related to their $\mathrm{T}_{\mathrm{S}}$.

\section{AFD synaptic transmission causes calcium transients in AIY axons}

The AIY interneuron is the major recipient of AFD chemical synaptic output. We sought to measure AFD-evoked AIY activity by expressing cameleon in AIY with the cell-specific promoter of the $t t x-3$ gene. We exposed immobilized worms to sinusoidal temperature oscillations added to positive linear ramps, which leads to strongly phase-locked activity of AFD in its operating range. Interestingly, we were unable to measure $\mathrm{Ca}^{2+}$ dynamics in the AIY soma caused by these temperature variations (data not shown). However, we were able to measure $\mathrm{Ca}^{2+}$ dynamics in a varicosity of the AIY axon near its joint with the nerve ring, which according to electron microscopic reconstruction of AIY is located near AFD postsynapses (White et al., 1985). We found that $\mathrm{Ca}^{2+}$ dynamics measured at the AIY axon also phase-locks to sinusoidal stimulus waveforms, but with different temporal characteristics than the phase-locking of AFD intracellular $\mathrm{Ca}^{2+}$ dynamics. The $\mathrm{Ca}^{2+}$ response at the AIY axon peaks sharply as soon as the sinusoidal waveform enters the warming phase and then falls to baseline, leading to a sawtooth response (Fig. 6A). The lower bound of temperatures that evoked AIY $\mathrm{Ca}^{2+}$ dynamics matches the operating range of AFD (Fig. 6B). Direct evidence that $\mathrm{Ca}^{2+}$ dynamics at the AIY axons are caused by AFD synaptic output was provided by femtosecond laser ablation: destroying the soma of the ipsilateral AFD neuron with laser pulses invariably abolished $\mathrm{Ca}^{2+}$ dynamics in the AIY axon (Fig. 6C). These results indicate that AFD produces a burst of synaptic output that is tuned to the onset of each warming phase in an oscillating thermosensory signal.

\section{Discussion}

C. elegans thermotaxis is a complex, experience-dependent navigational behavior in which the worm exhibits an isothermal tracking mode at temperatures near its thermotactic set-point and a cryophilic mode at temperatures above its thermotactic set-point. To track isotherms without deviating by $>0.1^{\circ} \mathrm{C}$, the worm's thermosensory system must be able to detect the small temperature oscillations that accompany its zigzag movements
C
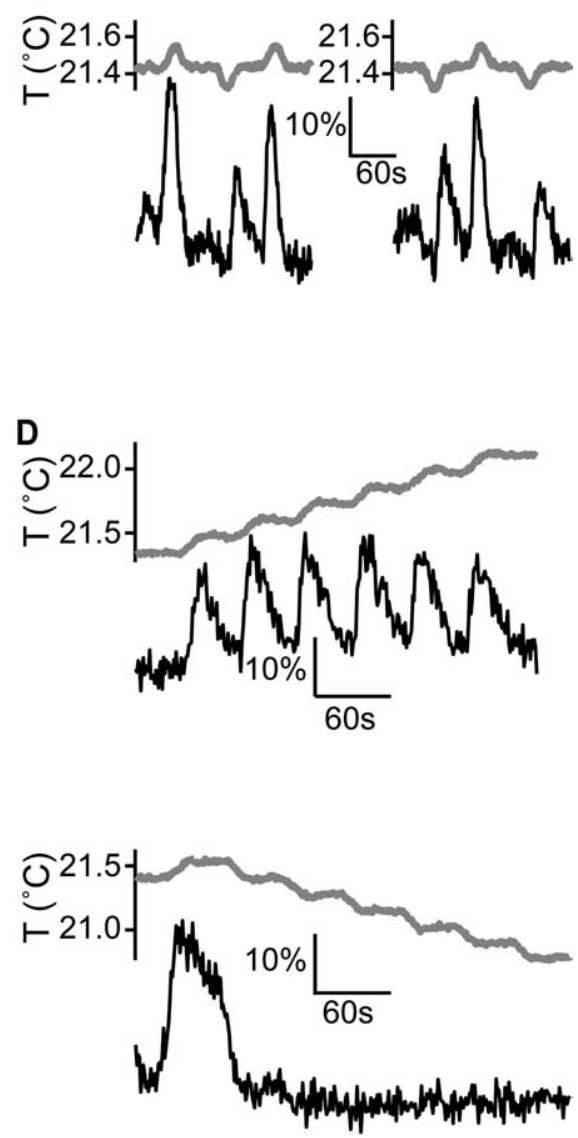

Figure 3. Responses to sine and step stimuli. $\boldsymbol{A}$, FRET measurements were made of the AFD soma responding to nonsaturating temperature sine waves within their operating range with 30 and 240 s periods (note the different scale bars for time). The gray lines show one cycle of the temperature waveforms, dashed lines show the representative FRET signal from one temperature cycle of a single worm, and the black lines show the mean FRET signals collected from 35 temperature cycles on 7 worms (for the 30 the mean response. $\boldsymbol{B}$, Worms were subjected to upsteps and downsteps in temperature, $0.2^{\circ} \mathrm{C}$ in size. The gray lines show one are shown at intervals along the mean response. $C$, Worms were subjected to positive and negative pulses, $0.1^{\circ} \mathrm{C}$ in amplitude

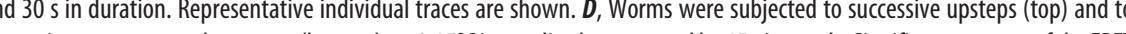
signal within $45 \mathrm{~s}$ after each upstep lead to a reliable response to the successive upstep. The FRET signal drops after the first downstep but does not recover significantly in $45 \mathrm{~s}$, and so no FRET signal is measured with subsequent downsteps.

on a spatial thermal gradient. To effect cryophilic movement, the worm has to be sensitive to positive and negative temperature changes over broad temperature ranges. Our study of AFD physiology has revealed that multiple properties that we attribute to the sophistication of C. elegans thermotaxis (long-term plasticity, high sensitivity to temperature gradients, and an ability to process thermosensory inputs) are all properties of one sensory neuronal type. In C. elegans, it appears that individual neurons at the sensory periphery may be endowed with complex sensory pathways, which might explain how a relatively simple neural circuit performs complex behavioral tasks. Below, we discuss each of the properties of the AFD neuron in turn.

The lower bound of the operating range of the AFD neuron reflects long-term plasticity by shifting to higher or lower temperatures based on the previous cultivation temperature. We have shown that the operating range of the AFD neuron is actually a property of its sensory endings; the sensory endings of 

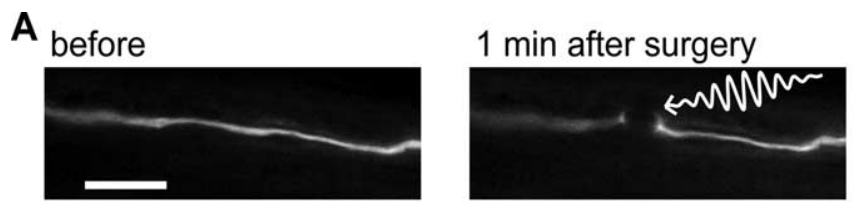

B

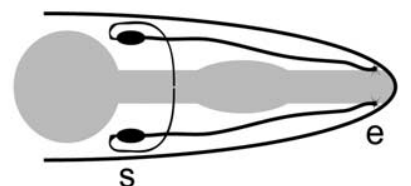

C
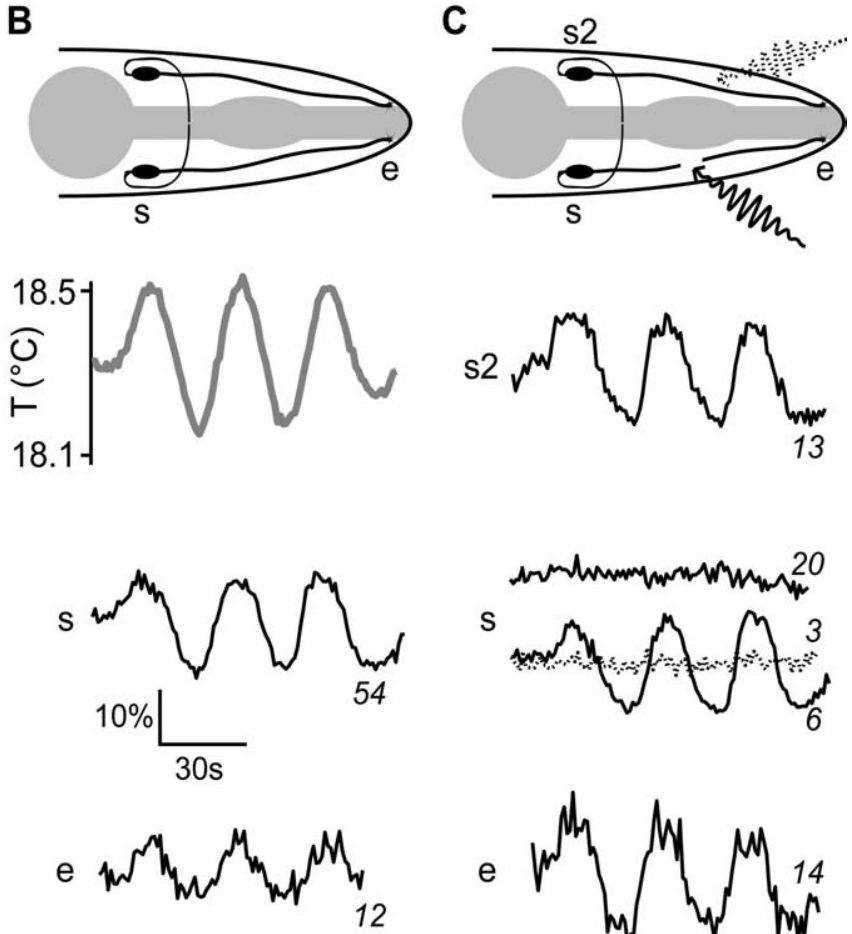

Figure 4. Spatial localization of FRET signal. $\boldsymbol{A}$, Representative images display the severing of an AFD dendrite using femtosecond laser pulses, showing the dendrite before surgery and $\sim 1$ min after surgery. Scale bar, $10 \mu \mathrm{m}$. $\boldsymbol{B}$, FRET measurements in response to a sinusoidal temperature stimulus (gray line) were taken at the soma of an AFD neuron (s) and the sensory endings of the AFD neuron (e). All worms in $\boldsymbol{B}$ and $\boldsymbol{C}$ were cultivated at $15^{\circ} \mathrm{C}$. $\boldsymbol{C}$, FRET measurements after severing one or both dendrites. In 13 cases in which we measured a FRET signal from the contralateral AFD soma (s2), its FRET signal was unaffected by severing the dendrite of its partner. In 20 of 26 cases in which an AFD dendrite was severed, the FRET signal was abolished in the parent soma (s). In the other 6 of the 26 cases in which an AFD dendrite was severed, a FRET signal persisted in the parent soma. In three of those six worms, we were also able to sever the dendrite of the contralateral AFD neuron, which in all three cases abolish the FRET signal in the soma (broken line in s). In 14 cases in which we measured a FRET signal from the sensory endings (e), the FRET signal was unaffected by severing the dendrite.

severed dendrites exhibit the same operating range as unsevered neurons. One possibility is that exposing C. elegans to new cultivation temperatures gradually resets the operating range of the thermoreceptor(s), which in turn, resets the operating range of AFD synaptic output, thereby resetting the temperature ranges of isothermal tracking and cryophilic movement. Thus, long-term plasticity in the thermosensory system of $C$. elegans seems to be encoded in part by an early event in the thermosensory pathway, such that the thermotactic set-point is retained by the sensory endings of a severed AFD dendrite.

We have shown that the AFD neuron is sensitive to temperature changes as small as $0.05^{\circ} \mathrm{C}$ and is also able to reset its sensitivity by adaptive processes. After sudden temperature changes, the level of intracellular $\mathrm{Ca}^{2+}$ in AFD passively returns to its prestimulus level, although it is interesting to note that adaptation after temperature downsteps is slower than adaptation after temperature upsteps. Adaptation explains how the AFD neuron is able to maintain exquisite temperature sensitivity over wide
A
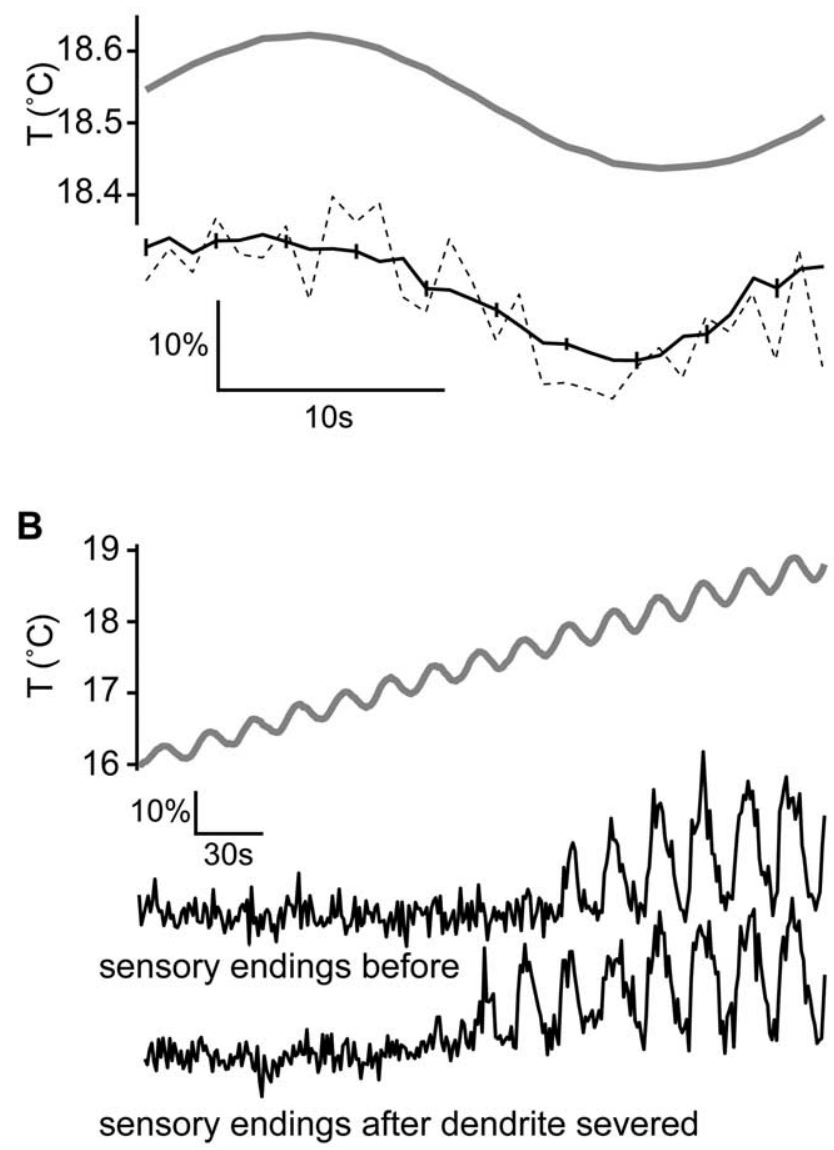

C

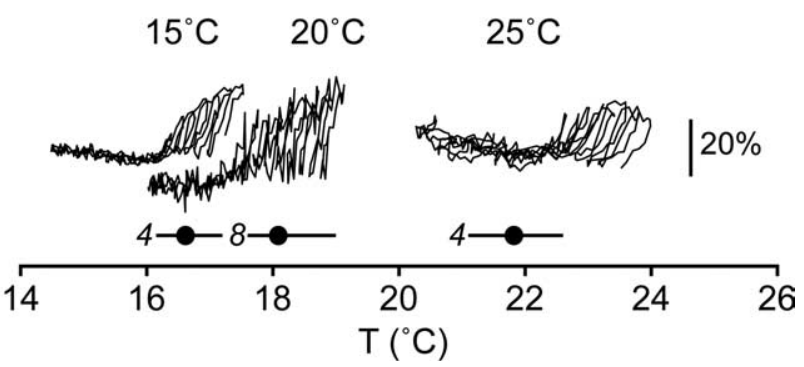

Figure 5. Calcium dynamics in the sensory endings. $\boldsymbol{A}$, FRET signals from the sensory endings of an unsevered $A F D$ dendrite were measured in response to a sinusoidal temperature stimulus, with $0.2^{\circ} \mathrm{C}$ peak-to-peak amplitude and 30 s period. The thick black line shows the average FRET signal from 30 stimulus cycles on nine worms cultivated at $15^{\circ} \mathrm{C}$; the dashed line shows a representative response from one cycle of one worm. The 1 SEM error bars show the average FRET signal. $B, A 20$ s sine wave added to a positive linear ramp was used to detect the existence of a threshold temperature in the AFD sensory endings after the dendrite was severed from its cell body. The sensory endings continued to exhibit an activity threshold correlated with $\mathrm{T}_{\mathrm{s}}$. The top trace shows the response of the sensory endings of an unsevered dendrite, whereas the bottom trace shows the response of the sensory endings after severing the dendrite. This worm was cultivated at $20^{\circ} \mathrm{C}$. In a few cases, such as the one shown, the activity threshold of the sensory endings would shift upward or downward by as much as $1^{\circ} \mathrm{C}$ after severing the dendrite. $C$, The activity threshold temperature was measured for worms cultivated at 15,20 , and $25^{\circ} \mathrm{C}$, as in Figure $1 \mathrm{C}$. For each cultivation temperature, sample parametric plots of FRET versus temperature are shown; the circle represents the mean value of the measurements of activity threshold, the line represents the full range of the measurements, and the number of worms tested is shown in italics.

operating ranges. But the bidirectional response (temperature increases and decreases actively raise and lower the level of intracellular $\mathrm{Ca}^{2+}$ in AFD) suggests that AFD is also actively reset by temperature changes of opposite sign. The bidirectional response 


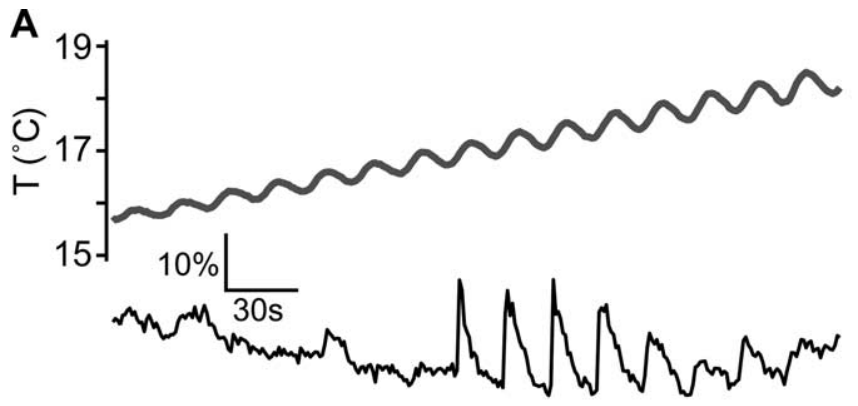

B
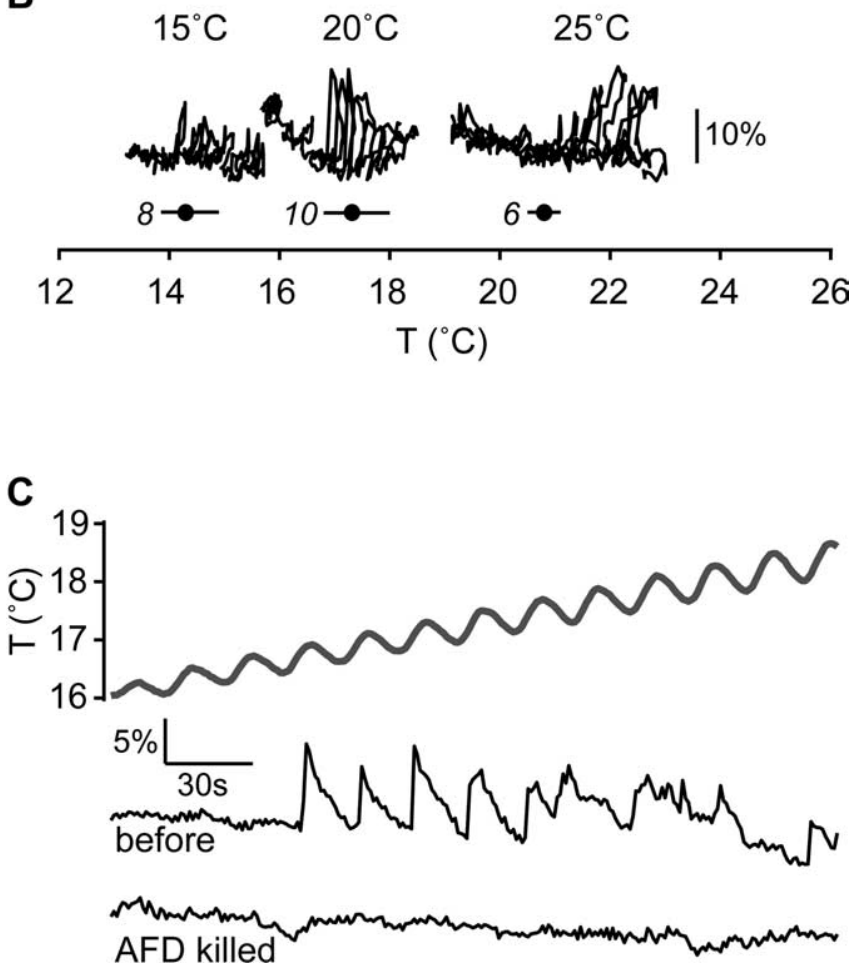

Figure 6. Postsynaptic $\mathrm{Ca}^{2+}$ dynamics in the AIY axon. A, Worms with wild-type background expressing cameleon in AIY were subjected to temperature stimuli consisting of a small sine wave added to a linear ramp, as in Figure $1 C$. We recorded FRET signals from a varicosity of the AIY axon near the ventral edge of the nerve ring. The FRET signal from AIY phase-locks to the sinusoidal oscillation, but only above a threshold temperature comparable to the threshold temperature of AFD activity (see Fig. 1C). B, As in Figure 1C, we show a representative FRET signal from an AlY exhibiting a threshold temperature for a worm cultivated $20^{\circ} \mathrm{C}$. Representative parametric plots of the FRET signal versus temperature of individual worms cultivated at 15 , 20 , and $25^{\circ} \mathrm{C}$ are shown. Below each representative parametric plot, we show the activity threshold temperature corresponding to each cultivation temperature. For each cultivation temperature, the circle represents the mean value of the measurements of activity threshold, the line represents the full range of the measurements, and the number of worms tested is shown in italics. C, To test whether the signal in AIY could be attributed to AFD synaptic output, we subjected worms to the stimulus in Figure $5 A$, killed the ipsilateral AFD soma with femtosecond laser pulses, and repeated the stimulus. In 10 of 10 surgeries, killing AFD eliminated the FRET signal in the second run. In mock surgery experiments, in which the experimental protocol was the same, except that AFD was not irradiated with femtosecond laser pulses, only in 2 of 13 cases did we not measure a phase-locked FRET signal in the second run.

explains why the level of intracellular $\mathrm{Ca}^{2+}$ in AFD is able to robustly phase-lock to each warming and cooling phase of oscillatory temperature waveforms.

We have also shown that different sensory transduction events in the AFD neuron lock to different phases of an oscillatory temperature waveform. A burst of AFD chemical synaptic transmission, the spike in intracellular $\mathrm{Ca}^{2+}$ levels in the AIY axon, is triggered by the onset of each warming phase and is then rapidly turned off. The level of intracellular $\mathrm{Ca}^{2+}$ in AFD peaks shortly thereafter. Intracellular $\mathrm{Ca}^{2+}$ in AFD is likely to play a direct role in the biochemistry of AFD sensory transduction and might contribute to shaping the time course of the physiological response. One possibility is that the onset of each warming phase stimulates AFD synaptic output and the delayed rise in the level of AFD intracellular $\mathrm{Ca}^{2+}$ contributes to shutting off the thermosensory response and synaptic output; the cooling phase clears intracellular $\mathrm{Ca}^{2+}$, resets the AFD neuron, and prepares the response to the next warming phase. In fact, a role for $\mathrm{Ca}^{2+}$ in short-term adaptation and response turn-off is a nearly universal property of sensory systems (Fain et al., 2001; Holt and Corey, 2000; Zufall and Leinders-Zufall, 2000).

The operating range of AFD spans the temperature range of isothermal tracking and of cryophilic movement, and we infer that AFD might have a role in mediating one or both of these behaviors. It appears that the sensory pathways in the AFD neuron enhance the ability of AFD to detect each cycle of an oscillatory thermosensory input. Detecting an oscillating temperature waveform has direct behavioral relevance, because such an oscillatory input would accompany the zigzag movements of a worm during isothermal tracking. This is consistent with the observations that genetic or physical perturbation of AFD functions abolish isothermal tracking (Mori and Oshima, 1995; Satterlee et al., 2001; Ryu and Samuel, 2002). For example, during isothermal tracking on spatial thermal gradients with $1^{\circ} \mathrm{C} / \mathrm{cm}$ steepness, the side-to-side movements of the worm's nose, and thereby the side-to-side movements of the AFD thermosensors, will drive sinusoidal temporal oscillations with $\sim 0.05^{\circ} \mathrm{C}$ amplitude, which is comparable to our measurements of the physiological sensitivity of the AFD neuron. When crawling directly up or down these spatial thermal gradients at typical crawling speeds during cryophilic movement, C. elegans will encounter linear temporal gradients of $0.02^{\circ} \mathrm{C} / \mathrm{s}$, which is also well within the range of AFD sensitivity.

An outstanding question in C. elegans neurobiology is how relatively simple and even overlapping neural circuits drive the variety of complex sensorimotor behaviors exhibited by the worm. Here, we have shown that one sensory neuronal type encodes many of the functions specific to thermotactic navigation, a particularly complex, experience-dependent navigational behavior. This observation suggests that $C$. elegans may generate sophisticated behaviors by sophisticated computational analyses performed by individual neurons at the sensory periphery.

\section{References}

Brenner S (1974) The genetics of Caenorhabditis elegans. Genetics 77:71-94. Chalfie M, Sulston JE, White JG, Southgate E, Thomson JN, Brenner S (1985) The neural circuit for touch sensitivity in Caenorhabditis elegans. J Neurosci 5:956-964.

Chung SH, Clark DA, Gabel CV, Mazur E, Samuel AD (2006) The role of the AFD neuron in C. elegans thermotaxis analyzed using femtosecond laser ablation. BMC Neurosci 7:30.

Coburn CM, Bargmann CI (1996) A putative cyclic nucleotide-gated channel is required for sensory development and function in C. elegans. Neuron 17:695-706.

Fain GL, Matthews HR, Cornwall MC, Koutalos Y (2001) Adaptation in vertebrate photoreceptors. Physiol Rev 81:117-151.

Furshpan EJ, Furukawa T (1962) Intracellular and extracellular responses of the several regions of the Mauthner cell of the goldfish. J Neurophysiol 25:732-771. 
Gray JM, Hill JJ, Bargmann CI (2005) A circuit for navigation in Caenorhabditis elegans. Proc Natl Acad Sci USA 102:3184-3191.

Hedgecock EM, Russell EL (1975) Normal and mutant thermotaxis in the nematode Caenorhabditis elegans. Proc Natl Acad Sci USA 72:4061-4065.

Hobert O, Mori I, Yamashita Y, Honda H, Ohshima Y, Liu Y, Ruvkun G (1997) Regulation of interneuron function in the C. elegans thermoregulatory pathway by the ttx-3 LIM homeobox gene. Neuron 19:345-357.

Holt JR, Corey DP (2000) Two mechanisms for transducer adaptation in vertebrate hair cells. Proc Natl Acad Sci USA 97:11730-11735.

Kerr R, Lev-Ram V, Baird G, Vincent P, Tsien RY, Schafer WR (2000) Optical imaging of calcium transients in neurons and pharyngeal muscle of C. elegans. Neuron 26:583-594.

Kimura KD, Miyawaki A, Matsumoto K, Mori I (2004) The C. elegans thermosensory neuron AFD responds to warming. Curr Biol 14:1291-1295.

Komatsu H, Mori I, Rhee JS, Akaike N, Ohshima Y (1996) Mutations in a cyclic nucleotide-gated channel lead to abnormal thermosensation and chemosensation in C. elegans. Neuron 17:707-718.

Miyawaki A, Griesbeck O, Heim R, Tsien RY (1999) Dynamic and quantitative $\mathrm{Ca}^{2+}$ measurements using improved cameleons. Proc Natl Acad Sci USA 96:2135-2140.

Mori I, Ohshima Y (1995) Neural regulation of thermotaxis in Caenorhabditis elegans. Nature 376:344-348.

Ryu WS, Samuel ADT (2002) Thermotaxis in Caenorhabditis elegans ana- lyzed by measuring responses to defined thermal stimuli. J Neurosci 22:5727-5733.

Satterlee JS, Sasakura H, Kuhara A, Berkeley M, Mori I, Sengupta P (2001) Specification of thermosensory neuron fate in C. elegans requires ttx- 1 , a homolog of otd/Otx. Neuron 31:943-956.

Sengupta P, Colbert HA, Bargmann CI (1995) The C. elegans gene odr-7 encodes an olfactory-specific member of the nuclear receptor superfamily. Cell 79:971-980.

Shen N, Datta D, Schaffer CB, LeDuc P, Ingber DE, Mazur E (2005) Ablation of cytoskeletal filaments and mitochondria in cells using a femtosecond laser nanoscissor. Mech Chem Biosys 2:17-26.

Tsalik EL, Hobert O (2003) Functional mapping of neurons that control locomotory behavior in Caenorhabditis elegans. J Neurobiol 56:178-197.

Wakabayashi T, Kitagawa I, Shingai R (2004) Neurons regulating the duration of forward movement in Caenorhabditis elegans. Neurosci Res 50:103-111.

White JG, Southgate E, Thomson JN, Brenner S (1986) The structure of the nervous system of Caenorhabditis elegans. Philos Trans R Soc Lond B Biol Sci 314:1-340.

Yanik MF, Cinar H, Cinar HN, Chisholm AD, Jin Y, Ben-Yakar A (2004) Neurosurgery: functional regeneration after laser axotomy. Nature 432:822.

Zufall F, Leinders-Zufall T (2000) The cellular and molecular basis of odor adaptation. Chem Senses 25:473-481. 